\title{
Artéria Coronária Única
}

\author{
Ênio Eduardo Guérios ${ }^{1,2,3}$, Paulo Maurício P. Andrade ${ }^{1,2}$, Gilberto Melnick ${ }^{1,2,3}$, Dirceu S. Barbosa Neto ${ }^{4}$
}

\section{RESUMO}

Artéria coronária única é uma anomalia coronária congênita extremamente rara. Embora não seja identificada com muita frequência, por apresentar, em geral, pouca repercussão clínica, a anomalia pode ser causa de isquemia miocárdica significativa e morte súbita. Relatamos o caso de uma paciente de 80 anos de idade, atendida em fase aguda de infarto agudo do miocárdio, que teve o diagnóstico de artéria coronária única durante o cateterismo que precedeu a angioplastia coronária primária. Segue-se uma revisão da literatura, abordando a classificação dessa anomalia, sua fisiopatologia, manifestações e significância clínica, e abordagens diagnósticas e terapêuticas.

DESCRITORES: Vasos coronários. Anormalidades congênitas. Infarto do miocárdio. Revisão.

A nomalias de artérias coronárias têm prevalência de cerca de $1,3 \%$ na população geral, encontrando-se na literatura uma variação entre $0,2 \%$ e 5,6\%. ${ }^{1}$ Na maioria dos casos, essas anomalias são achados isolados, sem repercussão clínica ou hemodinâmica. Entretanto, podem se associar a outros defeitos cardíacos, como valvopatias (valva aórtica bicúspide) e doenças cardíacas congênitas (tetralogia de Fallot ou transposição dos grandes vasos da base), e podem ser causa de isquemia miocárdica e morte súbita. ${ }^{2}$

A artéria coronária única é uma entidade extremamente rara, correspondendo de $2 \%$ a $4 \%$ de todas as anomalias coronárias, e sendo encontrada em apenas $0,0024 \%$ a $0,066 \%$ das coronariografias. Nesses casos, apenas uma artéria coronária se origina da aorta ascendente, e é responsável pelo suprimento sanguíneo de todo o coração. ${ }^{3}$ Descrevemos neste artigo o caso de uma paciente com artéria coronária única,

\footnotetext{
1 Serviço de Hemodinâmica - Hospital Santa Cruz - Curitiba, PR, Brasil.

2 Hospital Pilar - Curitiba, PR, Brasil.

3 Hospital de Clínicas da UFPR - Curitiba, PR, Brasil.

${ }^{4}$ Serviço de Radiologia - Hospital Santa Cruz - Curitiba, PR, Brasil.

Correspondência: Ênio Eduardo Guérios. Avenida Batel, 1.889 -

Curitiba, PR, Brasil - CEP 80420-090

E-mail: eguerios@terra.com.br

Recebido em: 17/3/2010 • Aceito em: 31/5/2010
}

\section{ABSTRACT}

\section{Single Coronary Artery}

Single coronary artery is an extremely rare congenital coronary anomaly. Although it is not frequently identified, due to the lack of clinical manifestations, it may cause significant myocardial ischemia and sudden death. We report the case of an 80-year old woman who had an acute myocardial infarction and was found to have a single coronary artery during primary coronary angioplasty. A literature review, detailing the classification, pathophysiology, clinical profile and significance, screening and therapeutic approaches of this anomaly is provided.

KEY-WORDS: Coronary vessels. Congenital abnormalities. Myocardial infarction. Review.

internada em decorrência de infarto agudo do miocárdio e tratada com angioplastia coronária primária.

\section{RELATO DO CASO}

Paciente do sexo feminino, com 80 anos de idade, foi internada em decorrência de quadro súbito de dor epigástrica e retroesternal, de forte intensidade, com cerca de 60 minutos de duração, com início poucas horas após ter realizado colonoscopia. Chegou ao pronto-atendimento já sem dor. Relatava história de hipertensão arterial e artrite reumatoide, ambas compensadas e em tratamento regular. Foi tabagista moderada por mais de 40 anos, tendo parado de fumar há 20 anos. Apresentava, na admissão, pressão arterial de 132/60 mmHg, pulso = frequência cardíaca de 58 bpm, e exame físico segmentar sem alterações significativas.

O eletrocardiograma realizado na admissão demonstrava apenas a presença de bradicardia sinusal e distúrbio de condução do ramo direito do feixe de His. As enzimas cardíacas à admissão, creatina quinase fração $\mathrm{MB}(\mathrm{CK}-\mathrm{MB})=30 \mathrm{U} / \mathrm{l}$ (valor de referência $<16 \mathrm{U} / \mathrm{l})$ e troponina I =0,61 ng/ml (valor de referência $<0,4 \mathrm{ng} / \mathrm{ml}$ ), subiram, respectivamente, para $42 \mathrm{U} / \mathrm{l}$ e 5,28 ng/ml após três horas.

Definido o quadro de infarto agudo do miocárdio sem supradesnivelamento de ST, a paciente recebeu ácido acetilsalicílico e dose de ataque $(600 \mathrm{mg})$ de 
clopidogrel, e foi encaminhada ao cateterismo cardíaco de urgência. Na coronariografia, observou-se uma artéria coronária direita de grande dominância, tortuosa, com obstrução de 50\% em seu terço médio, seguida por outra de $95 \%$ em seu terço distal, considerada a lesão culpada pelo quadro agudo. O tronco da artéria coronária esquerda se originava a partir do terço inicial da artéria coronária direita, tendo um trajeto anterior até o terço médio do sulco interventricular, de onde nutria os ramos descendente anterior e circunflexo, com topografias normais e sem lesões obstrutivas (Figura 1). A ventriculografia esquerda evidenciou discreta hipocinesia de paredes ínfero-basal e ínfero-medial, com fração de ejeção preservada (74\%).

Procedeu-se à realização de angioplastia primária da coronária direita com implante de stent convencional, com bom resultado imediato (Figura 2). A paciente teve boa evolução hospitalar, e recebeu alta no quinto dia de evolução, em bom estado geral.

Após seis meses, ainda assintomática, a paciente realizou angiotomografia coronária de controle. Compro- vou-se o trajeto pré-pulmonar do tronco da coronária esquerda, e evidenciou-se a boa evolução do stent implantado na fase aguda do infarto, com discreta redução luminal (Figuras 3 e 4).

\section{DISCUSSÃO}

As anomalias coronárias podem ser classificadas de duas formas. A primeira leva em conta suas características anatômicas, e as divide em anomalias de origem ou curso (óstio ectópico no próprio seio coronário, origem fora do seio coronário normal, atresia coronária), anomalias de anatomia intrínseca (estenose congênita, aneurismas congênitos, ponte miocárdica) e anomalias de terminação (fístulas coronárias). ${ }^{1,4} \mathrm{~A}$ outra classificação se baseia no potencial de a anomalia em questão ser causa de isquemia miocárdica: anomalias coronárias maiores são aquelas que podem causar isquemia miocárdica, como fístula coronária, origem anômala da artéria coronária esquerda a partir da artéria pulmonar, artéria coronária única, e coronária com origem anômala a partir do seio contralateral e
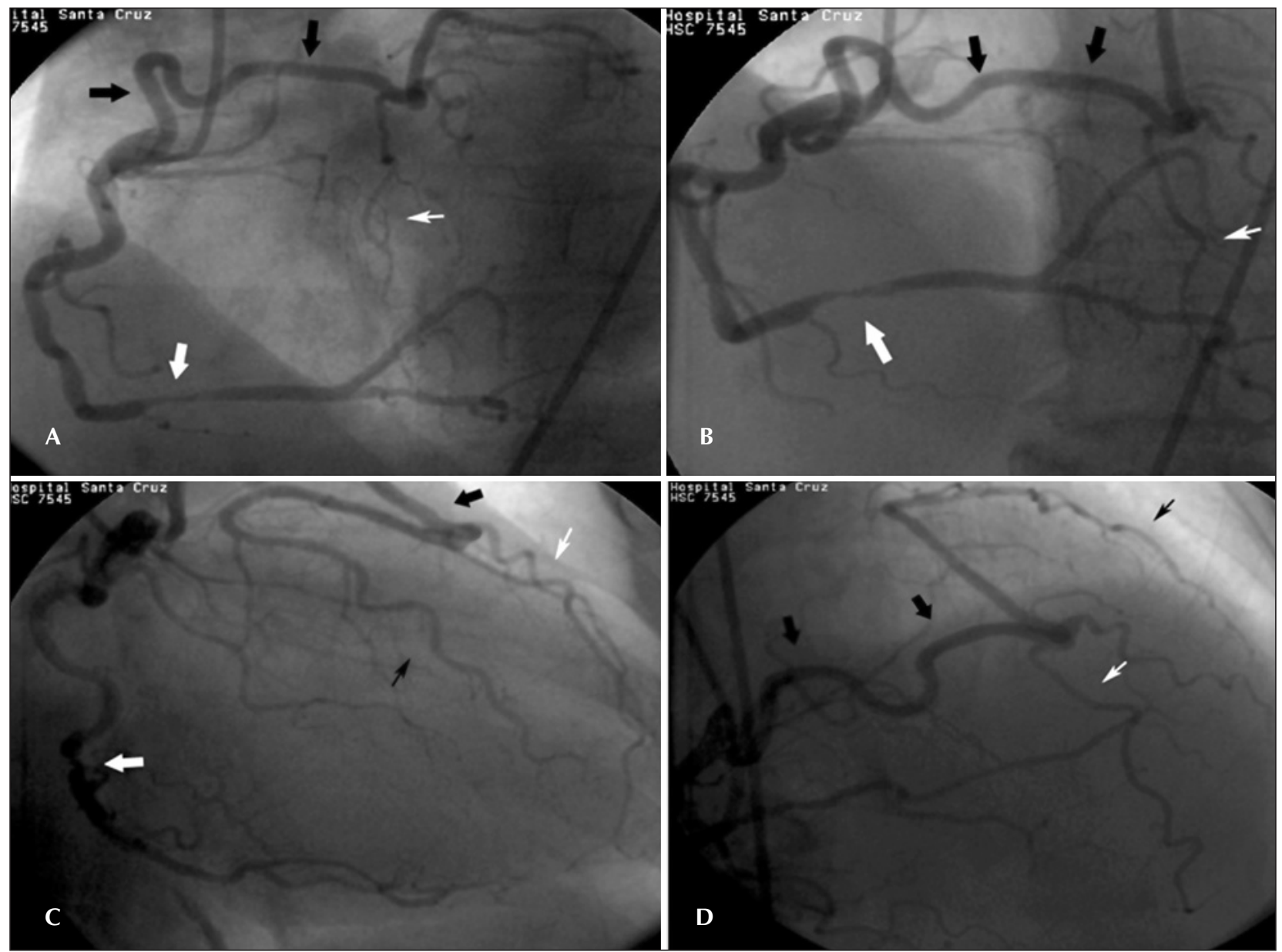

Figura 1 - Coronariografias em oblíqua anterior esquerda (A), oblíqua anterior esquerda cranial (B), oblíqua anterior direita (C) e póstero-anterior cranial (D). Seta escura grossa = tronco da coronária esquerda com trajeto anômalo; seta escura fina = ramo circunflexo; seta branca grossa = lesão culpada pelo infarto, na coronária direita; seta branca fina = ramo descendente anterior. 
curso interarterial; anomalias menores são aquelas que não causam isquemia miocárdica, como óstios ectópicos no próprio seio coronário, artéria circunflexa com origem no seio coronário direito, e duplicação arterial coronária. ${ }^{5}$

A primeira descrição de artéria coronária única foi feita por Thebesius, em 1716. ${ }^{6}$ Em 1950, um estudo de 1.895 autópsias revelou prevalência de artéria coronária única em 0,037\% da população normal ${ }^{7}$, e em 1967 Halperin, Penny e Kennedy ${ }^{8}$ relataram o primeiro diagnóstico angiográfico dessa anomalia. Em uma meta-análise compreendendo quase 240 mil angiografias coronárias, estabeleceu-se prevalência de artéria coronária única de 0,044\%.9 Não há predominância de origem da artéria coronária única a partir do seio direito ou esquerdo de Valsalva, mas a anomalia foi reportada mais frequentemente em homens. Relata-se que $40 \%$ dos pacientes com artéria coronária única apresentam

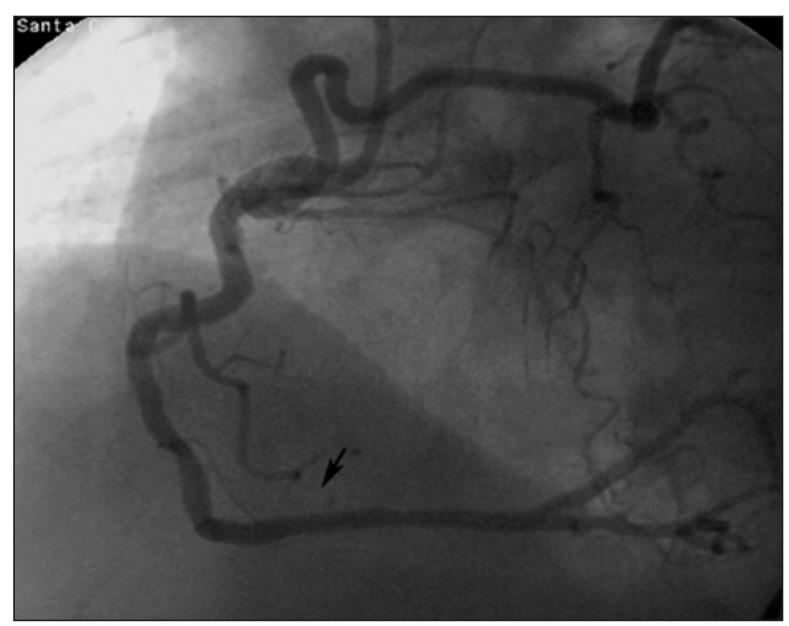

Figura 2 - Resultado final da angioplastia primária com implante de stent em coronária direita. outras cardiopatias congênitas associadas, como tetralogia de Fallot, transposição dos grandes vasos, truncus arteriosus, fístulas arteriovenosas coronárias e valva aórtica bicúspide. ${ }^{3,4}$

Lipton et al. ${ }^{10}$ sugeriram uma classificação angiográfica da artéria coronária única, posteriormente modificada por Yamanaka e Hobbs ${ }^{11}$ (Figura 5). De acordo com essa proposta, a artéria coronária única é classificada em dois grupos, segundo a localização de seu óstio - "R", se a partir do seio coronário direito, e "L", se a partir do esquerdo. Esses grupos se subdividem em tipos I, II e III, conforme a distribuição anatômica do vaso. No grupo I, um vaso único segue o curso normal de uma artéria coronária direita (R-I) ou esquerda (L-I), e se continua, via colaterais, para nutrir o território da coronária contralateral. No grupo II, uma coronária se origina na porção proximal da coronária contralateral, que tem origem normal, e atravessa a base do coração, até atingir sua distribuição normal. Esse grupo se subdivide em cinco subgrupos: A, se o trajeto da artéria é anterior aos grandes vasos da base; B, se entre aorta e artéria pulmonar; $\mathrm{P}$, se posterior aos grandes vasos da base; $S$, se cursa através do septo interventricular; e C, que inclui qualquer combinação dessas rotas. Finalmente, no grupo III, o ramo descendente anterior e o ramo circunflexo nascem separadamente na parte proximal de uma artéria coronária direita normal (R-III). O caso da paciente descrita neste artigo se encaixa na classificação R-II-A.

Em cerca de $85 \%$ dos portadores de artéria coronária única a anomalia é benigna, os pacientes são assintomáticos, e o diagnóstico é estabelecido como achado fortuito em uma coronariografia de rotina ou emergencial, como no caso de nossa paciente. Entretanto, $15 \%$ dos pacientes podem apresentar isquemia miocárdica como consequência direta da anatomia coronária anormal, independentemente de doença arterial coronária. ${ }^{3}$ De fato, anomalias arteriais coronárias
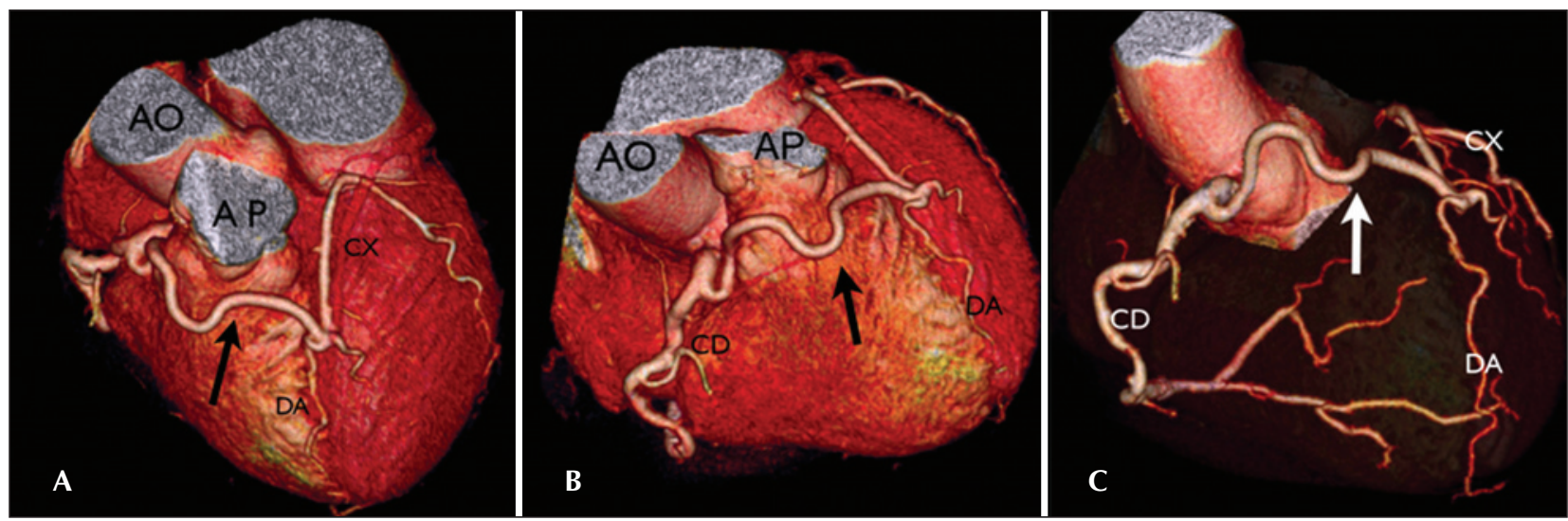

Figura 3 - Imagens da tomografia por multidectores. Reconstruções em 3D da coronária esquerda com origem no segmento proximal da artéria coronária direita e trajeto pré-pulmonar. Seta = tronco da coronária esquerda com trajeto anômalo. $\mathrm{AO}=$ aorta; $\mathrm{AP}=$ artéria pulmonar; $\mathrm{CD}=$ artéria coronária direita; $\mathrm{CX}=$ artéria circunflexa; $\mathrm{DA}=$ artéria descendente anterior. 
são a segunda causa de morte súbita em atletas jovens. ${ }^{2,12} \mathrm{Em}$ um estudo de uma série de 6,3 milhões de recrutas americanos submetidos a oito semanas de treinamento intensivo, Eckart et al. ${ }^{13}$ identificaram 277 óbitos não relacionados a trauma. Das 64 mortes de origem cardíaca, 21 (33\%) decorreram de origem anômala de artéria coronária esquerda a partir do seio coronário direito. Nenhuma outra anomalia coronária foi causa de óbito nessa população. ${ }^{13}$ Em comparação, Drory et al. ${ }^{14}$ relataram uma série contínua de 162 autópsias de pacientes jovens $(<40$ anos) que

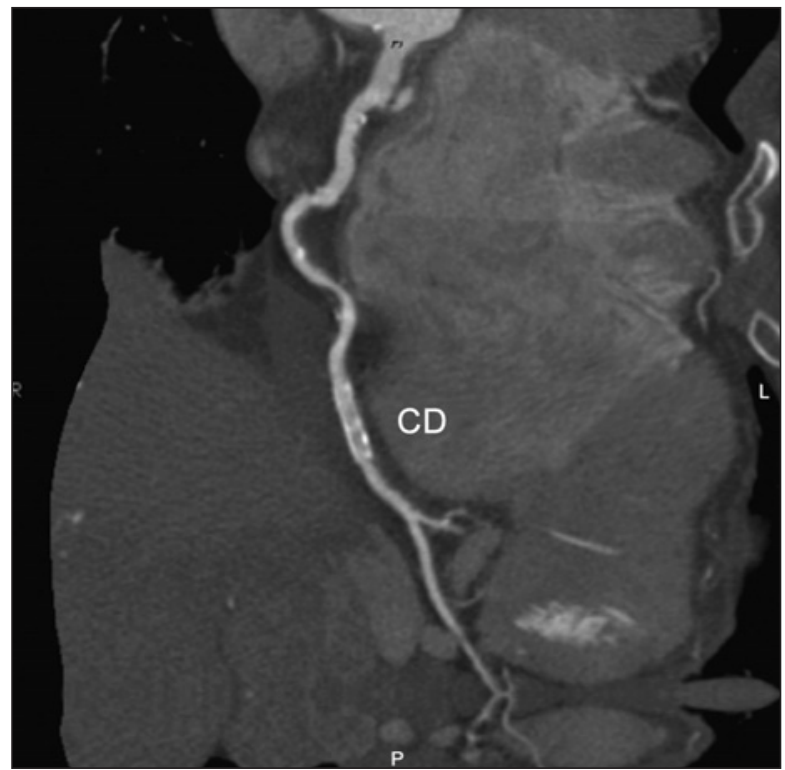

Figura 4 - Controle tardio da angioplastia primária pela tomografia por multidectores. Imagens multiplanares com reconstrução em curva da artéria coronária direita (CD), mostrando endoprótese no segmento distal com proliferação neointimal de grau discreto. tiveram morte súbita. Destas, apenas uma $(0,6 \%)$ foi relacionada a anomalia coronária. Confrontando-se os resultados desses dois trabalhos, pode-se sugerir que o exercício físico intenso desempenha papel importante como causa de óbito em pacientes com essas anomalias.

Estudos anatomopatológicos sugerem que, em pacientes com isquemia determinada por artéria coronária única, a restrição de fluxo coronário ocorre em vários surtos, infrequentes porém cumulativos, resultando em ilhas de necrose e fibrose no miocárdio. Essas alterações histopatológicas podem ser substrato de instabilidade elétrica, e predispor a taquiarritmias potencialmente letais. $^{2}$

Várias teorias tentam explicar a gênese da isquemia miocárdica nesses pacientes. Um dos fatores implicados é o ângulo agudo formado pela origem do vaso anômalo, determinando um óstio em forma de fenda que pode ser comprimido na sístole. Outro fator considerado é a compressão do vaso anômalo que tenha curso interarterial (artéria coronária única do tipo R-II-B ou L-II-B) pela aorta e artéria pulmonar, especialmente durante exercício físico, quando esses vasos sofrem dilatação.

Questiona-se, entretanto, o papel da compressão extrínseca pelos grandes vasos como mecanismo causador de isquemia miocárdica em pacientes portadores de artérias coronárias únicas do subgrupo II-B, uma vez que a pressão hidrostática da artéria pulmonar é muito inferior à pressão de perfusão coronária. ${ }^{8}$ Baseado em estudos com ultrassom intracoronário, Angelini ${ }^{15,16}$ demonstrou consistentemente que, nessas anomalias, a artéria coronária ectópica apresenta, logo após sua origem, um trajeto intramural, ou seja, tem intussuscepção na parede da aorta. Esse é justamente o local de maior proximidade entre aorta e artéria pulmonar, reforçando a improbabilidade do mecanismo de com-

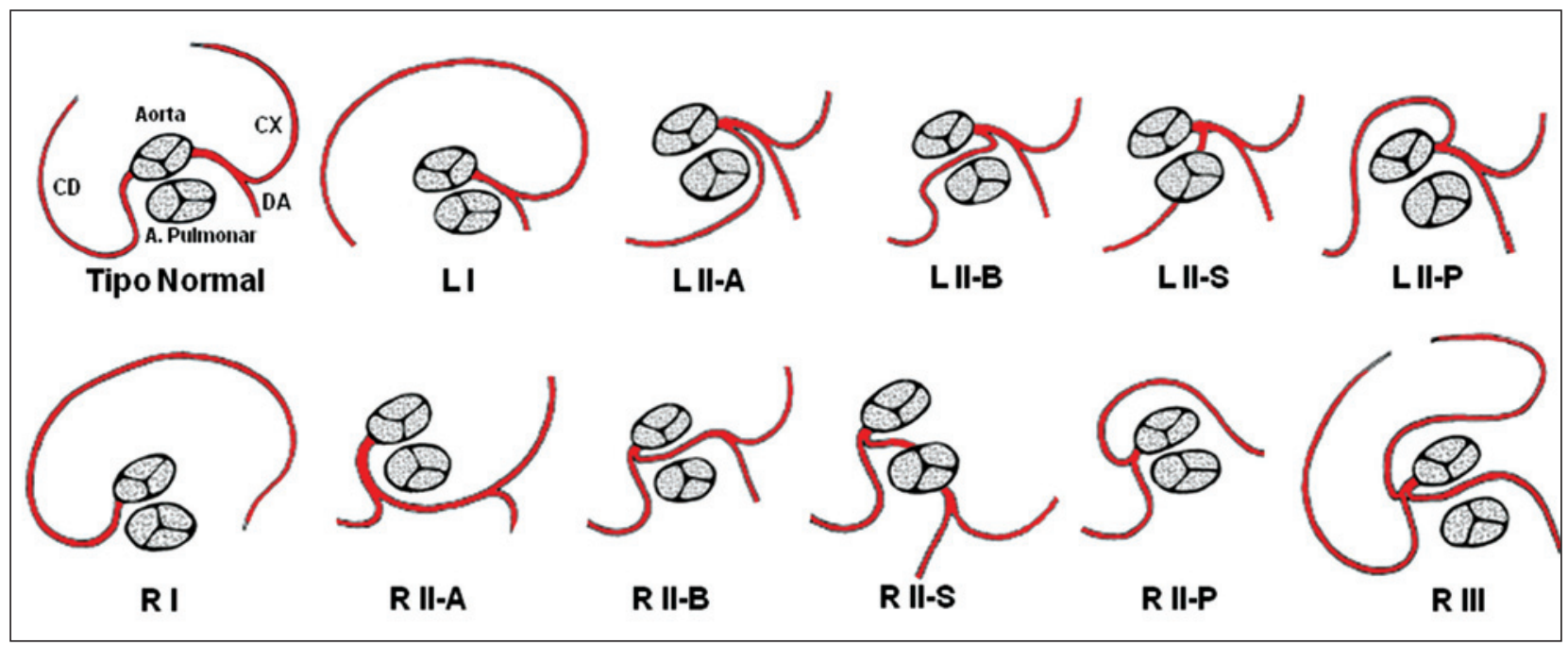

Figura 5 - Classificação angiográfica de artéria coronária única (ilustração baseada nas referências 1, 8, 10 e 11). 
pressão extrínseca. No entanto, nesse segmento, as paredes aórticas interna e externa ao vaso intramural são delgadas, permitindo compressão luminal fixa em todo o ciclo cardíaco, porém com exacerbação fásica durante a sístole. Estudos preliminares desse autor sugerem ainda haver variações individuais no grau de compressão sistólica, o que explica os diferentes comportamentos clínicos e prognósticos. Fatores que afetem a complacência aórtica, como ectasia da aorta ascendente ou síndrome de Marfan, ou fatores que modifiquem a pressão aórtica, como insuficiência aórtica, podem também interferir no prognóstico. ${ }^{15,16}$

Não existe apresentação clínica característica de pacientes portadores de artéria coronária única. Como assinalado previamente, na maioria dos pacientes a anomalia é benigna e sem repercussão clínica. Mesmo entre pacientes que sofreram morte súbita, 55\% a 93\% não tiveram manifestação clínica prévia que sugerisse sua anomalia. Cerca de $10 \%$ dos pacientes, porém, podem apresentar dispneia, palpitações, tontura, síncope e angina. Enquanto morte súbita tipicamente se relaciona com jovens após realização de exercício físico intenso, os outros sintomas são mais característicos de pacientes adultos, e se associam ao surgimento de hipertensão arterial, sugerindo que o enrijecimento progressivo da parede aórtica é um fator de proteção de morte súbita nesses adultos. ${ }^{15,16}$

Basso et al. ${ }^{2}$ descreveram 27 casos de atletas jovens que tiveram morte súbita secundária a artéria coronária única do tipo II-B. Doze desses jovens (45\%) apresentaram algum tipo de sintoma (síncope ou dor torácica) ou tinham testes cardiovasculares não-invasivos até 24 meses antes do óbito. Todos os testes, incluindo eletrocardiograma de repouso e de esforço e ecocardiograma, foram normais nesses pacientes. ${ }^{2}$

A literatura sugere que sintomas como dispneia, síncope, angina de esforço ou morte súbita ressuscitada, especialmente em jovens, devam suscitar a pesquisa de anomalias coronárias, mesmo na presença de teste de esforço negativo para isquemia. Como o ecocardiograma direcionado permite uma avaliação confiável do local de origem das artérias coronárias direita e esquerda ${ }^{17}$, esses pacientes devem ser primeiramente investigados com ecocardiograma transtorácico ou transesofágico. Caso esse exame não evidencie as origens da coronária esquerda ou direita a partir de seus locais habituais, deve-se complementar a investigação com coronariografia, ressonância magnética ou tomografia computadorizada. ${ }^{2,5,15}$

Uma vez comprovada a presença desse tipo específico de anomalia, os pacientes devem ser afastados de atividade física competitiva. ${ }^{2}$ A administração de betabloqueadores é provavelmente tão efetiva quanto a restrição de atividade física nesses pacientes. Descreve-se ainda o possível papel da angioplastia coronária com implante de stents nas anomalias do tipo R-II-B. ${ }^{16}$ Cirurgia é recomendada para correção de artéria coro- nária única do tipo L-II-B, e pode ser realizada de quatro formas: revascularização convencional, reimplante da artéria ectópica na raiz da aorta, excisão da parede comum ("teto") entre a aorta e o vaso anômalo, ou criação de um novo óstio ao final do segmento intramural do vaso. Após o tratamento específico, os pacientes devem se reavaliados antes de serem liberados para atividade física intensa. ${ }^{2,16}$

\section{CONFLITO DE INTERESSES}

Os autores declararam inexistência de conflito de interesses relacionado a este manuscrito.

\section{REFERÊNCIAS}

1. Earls JP. Coronary artery anomalies. Tech Vasc Interv Radiol. 2006;9(4):210-7.

2. Basso C, Maron BJ, Corrado D, Thiene G. Clinical profile of congenital coronary artery anomalies with origin from the wrong aortic sinus leading to sudden death in young competitive athletes. J Am Coll Cardiol. 2000;35(6):1493-501.

3. Ackay A, Tuncer C, Batyraliev T, Gokce M, Eryonucu B, Koroglu S, et al. Isolated single coronary artery: a series of 10 cases. Circ J. 2008;72(8):1254-8.

4. Angelini P. Normal and anomalous coronary arteries: definitions and classification. Am Heart J. 1989;117(2):418-27.

5. Kang JW, Seo JB, Chae EJ, Jang YM, Do KH, Lee JS, et al. Coronary artery anomalies: classification and electrocardiogramgated multidetector computed tomographic findings. Semin Ultrasound CT MRI. 2008;29(3):182-94.

6. Thebesius AC. Dissertation medica de circulo sanguinis in cordo. Ludg Batav, JA: Langerak; 1716.

7. Alexander RW, Griffith GC. Anomalies of the coronary arteries and their clinical significance. Circulation. 1956;14(5):800-5.

8. Halperin IC, Penny JL, Kennedy RJ. Single coronary artery: antemortem diagnosis in a patient with congestive heart failure. Am J Cardiol. 1967;19(3):424-7.

9. Kuon E, Ropers D. Single coronary artery - a rarity in the catheterization laboratory: case report and current review. Can J Cardiol. 2004;20(6):647-51.

10. Lipton MJ, Barry WH, Obrez I, Silverman JF, Wexler L. Isolated single coronary artery: diagnosis, angiographic classification and clinical significance. Radiology. 1979;130(1):39-47.

11. Yamanaka O, Hobbs RE. Coronary artery anomalies in 126,595 patients undergoing coronary angiography. Cathet Cardiovasc Diagn. 1990;21(1):28-40.

12. Maron BJ. Sudden death in young athletes. N Engl J Med. 2003;349(11):1064-75.

13. Eckart RE, Scoville SL, Campbell CL, Shry EA, Stajduhar KC, Potter RN, et al. Sudden death in young adults: a 25-year review of autopsies in military recruits. Ann Intern Med. 2004; 141(11):829-34.

14. Drory $Y$, Turetz $Y$, Hiss Y, Lev B, Fisman EZ, Pines A, et al. Sudden unexpected death in persons less than 40 years of age. Am J Cardiol. 1991;68(13):1388-92.

15. Angelini P. Coronary artery anomalies - current clinical issues: definitions, classification, incidence, clinical relevance, and treatment guidelines. Tex Heart Inst J. 2002;29(4):271-8.

16. Angelini P. Coronary artery anomalies: an entity in search of an identity. Circulation. 2007;115(10):1296-305.

17. Pelliccia A, Spataro A, Maron BJ. Prospective echocardiographic screening for coronary artery anomalies in 1,360 elite competitive athletes. Am J Cardiol. 1993;72(12):978-9. 